Relations industrielles

Industrial Relations

\title{
Labour Relations and Court Review, par Philip Cutler, Montréal, Tundra Books, 1968, 332 pages.
}

\section{Nicole L’Heureux}

Volume 25, numéro 1, 1970

URI : https://id.erudit.org/iderudit/028110ar

DOI : https://doi.org/10.7202/028110ar

Aller au sommaire du numéro

Éditeur(s)

Département des relations industrielles de l'Université Laval

ISSN

0034-379X (imprimé)

1703-8138 (numérique)

Découvrir la revue

Citer ce compte rendu

L'Heureux, N. (1970). Compte rendu de [Labour Relations and Court Review, par Philip Cutler, Montréal, Tundra Books, 1968, 332 pages.] Relations industrielles / Industrial Relations, 25(1), 153-154. https://doi.org/10.7202/028110ar

Tous droits réservés @ Département des relations industrielles de l'Université Laval, 1970
Ce document est protégé par la loi sur le droit d'auteur. L’utilisation des services d'Érudit (y compris la reproduction) est assujettie à sa politique d'utilisation que vous pouvez consulter en ligne.

https://apropos.erudit.org/fr/usagers/politique-dutilisation/ 
plantation du socialisme intégral. Une autre aile moins radicale cherche à établir un parti politique, inspiré du parti travailliste anglais, qui s'appuierait sur la force combinée des travailleurs et des agriculteurs. Enfin, à l'extrême droite, pourrait-on dire, on trouve parmi les dirigeants syndicaux, les tenants d'un autre système qui tend à infiltrer au sein des partis politiques traditionnels des chefs ouvriers dans l'espoir d'obtenir ainsi des avantages immédiats selon la formule pratiquée aux Etats-Unis par la Fédération américaine du Travail.

Il s'ensuit deux conséquences. D'une part, le mouvement syndical, qui ne représentait d'ailleurs qu'une faible minorité des travailleurs dans une société globalement assez peu industrialisée et urbanisée, n'a pu, ainsi divisé, servir d'infrastructure à la formation d'un véritable parti travailliste malgré les efforts déployés par certains de ses dirigeants. D'autre part, quand il est advenu par exception que de tels partis ont pu s'approcher du pouvoir ou être invités à y participer, comme ce fut le cas en Ontario après la guerre, le mouvement syndical n'a pas manqué l'occasion de leur tomber dessus à bras raccourcis, de telle sorte que le travail était toujours à recommencer, qu'il arrivait même assez souvent que, au plan électoral, les travailleurs se battaient les uns contre les autres.

On ne peut pas dire cependant que cette action politique aberrante a été totalement infructueuse malgré son échec apparent. Au plan syndical, elle a permis d'obtenir graduellement des mesures législatives avantageuses; dans le domaine de l'action politique, pendant que les hommes de droite se réfugiaient sous l'aile des vieux partis et que ceux de gauche, fort peu nombreux à la vérité, s'isolaient dans le parti communiste, le groupe le plus nombreux allait donner son adhésion en 1932 au parti C.C.F. dont les chefs n'étaient pas des militants syndicaux, mais des intellectuels capables peutêtre avec le temps de réunir au sein d'une formation politique unique travailleurs, cultivateurs et gens de la classe moyenne, parti qui serait à l'abri des schismes d'autrefois.

C'est là, assurément, un résumé beaucoup trop schématique d'une étude qui repose sur une documentation remarquable et sur une analyse poussée des faits recueillis.
On en retiendra que les mouvements radicaux, qu'il s'agisse du travaillisme, du socialisme ou du syndicalisme, ont combattu avec vigueur les formations politiques traditionnelles malgré la faiblesse de leurs moyens d'action, l'insuffisance du nombre et la multitude des erreurs et des trahisons inévitables qui souvent engendraient un pessimisme dommageable.

$\mathrm{Du}$ point de vue historique, cette étude est de nature à aider quiconque veut connaître et comprendre le déroulement de la vie politique canadienne, car l'auteur a rassemblé dans cet ouvrage une véritable mine de renseignements qui ne pourront qu'être fort utiles à l'historien de la vie politique canadienne.

De son côté, le politicologue, qui prendra le temps d'approfondir cette analyse, y trouvera également de profitables leçons.

\section{André ROY}

\section{Labour Relations and Court Review, par} Philip Cutler, Montréal, Tundra Books, 1968, 332 pages.

Plusieurs ouvrages ont été publiés récemment sur les tribunaux administratifs. L'étude que nous présente le docteur Cutler, laquelle a d'ailleurs fait l'objet de sa thèse de doctorat, a l'intérêt de les considérer sous l'aspect du droit du travail et par là de combler un vide important. Il fait le tour de toute la question des conflits de juridiction entre le droit de regard du tribunal de droit commun et l'indépendance de la Commission des relations de travail, arbitres et conciliateurs.

Après avoir situé les brefs de prérogative tant au Canada que dans le droit québécois, il retrace avec clarté les diverses tentatives du législateur pour soustraire la Commission des relations de travail à la juridiction de surveillance et de contrôle de la Cour Supérieure, face à l'interprétation que chacune obtenait des tribunaux, jusqu'à la rédaction de l'article 33 du Code de procédure civile, sorte de compromis entre la fonction exécutive et judiciaire mais dont l'intention est plus évidente en matière de prohibition et de certiorari. Et il examine ensuite la portée des clauses privatives dans le Code du travail. 
L'auteur, dans sa deuxième partie, discute de la nécessité d'un certain contrôle judiciaire en certaines matières relevant des relations du travail. Se basant sur la constitution canadienne, sur les principes du droit écrit et de la justice naturelle, il rejette l'idée de soustraire d'une façon absolue le tribunal administratif au pouvoir de surveillance du tribunal de droit commun pour favoriser plutôt une intervention judiciaire dans des limites restreintes qui peuvent être différentes selon les secteurs du droit administratif.

Dans la troisième partie, on suit avec intérêt l'évolution de l'intervention étatique en matière de relations du travail jusqu'à la négociation collective obligatoire. L'auteur fait ressortir le rôle des tribunaux qui n'a pas toujours été négatif et qui est complémentaire dans la mise à exécution de la convention collective.

Confrontant, ensuite, la Commission des relations de travail, arbitres et conciliateurs avec une véritable indépendance dans l'exercice de leurs fonctions, il fait ressortir certaines faiblesses (les pressions dont ils sont l'objet et interventions gouvernementales et ministérielles) qui contribuent à gêner leur autonomie.

Enfin, l'auteur souligne l'élément important qu'est le facteur temps dans les matières relevant du Code du travail. A cause des besoins particuliers à la négociation collective, des moyens doivent $\hat{\mathrm{e}}$ tre trouvés pour éliminer les longs délais provoqués par la revision judiciaire des matières relevant du Code du travail. Il souligne, d'une part, les principales faiblesses de l'appareil judiciaire actuel qui est encore à la recherche d'une véritable doctrine d'un système de négociation collective auquel on reconnaît une certaine autorité sans qu'il soit besoin de reprendre constamment la discussion sur l'effet de la clause privative en matière de relations du travail. Il suggère en outre une simplification de la procédure qui remplacerait les brefs de prohibition, certiorari et mandamus par une procédure sommaire unique au même effet. Ses recommandations ont le mérite de respecter l'esprit de notre tradition judiciaire et par là d'être plus facilement acceptables et réalisables.

On doit souligner le grand souci de clarté de l'ouvrage du docteur Cutler dans son exposé et dans la présentation. Il est une contribution importante au droit du travail et un instrument de base qui sera très utile autant pour les avocats, les étudiants que pour tous ceux qui s'intéressent aux relations de travail.

Nicole L'HEUREUX

Deferment Policy in the Selective, by David R. Bradford, Princeton, N.J., Industrial Relations Section: Princeton University, 1969, 64 pages.

This brief study describes the American Selective Service policies, and focuses on the changes made to the Universal Military Training and Service Act (1951) which formed the framework of the present Military Selective Service Act (1967), specifically in regard to deferment policy and the changing needs for military manpower.

This paper undertakes an examination of the problems posed in the period $1960-67$, an analysis of the changes made in 1967, and an examination of an alternate system that tries to redress the defects. This work is an outgrowth of an earlier one the author, an economist, prepared as a consultant to the National Advisory Commission on Selective Service in 1966-67.

In the 1960's, a reassessment of the Selective Service System was prompted by a widespread dissatisfaction with its performance in terms of equity and certainty. The charge of inequity arose primarily from the relatively low rates of participation by men at the two extremes of the educational (and correspondingly economic) spectrum of society. The changes made in 1967 appear to limit or even eliminate the advaritageous treatment of the highly educated. However, the problem of draft uncertainly still remains.

The objectives of a selective service system should include 1) flexibility, 2) economy, 3) equity, 4) certainty, and particularly considered by the author, 5) public acceptance. Changing demands for military manpower have led to changes in deferments. Three of the most important deferments specifically authorized before the changes in 1967 were the deferment of 1) fathers, 2) students, and 3 ) on grounds of occupation. It was the student deferment that led to controversy, mostly on the grounds of inequity. 